\title{
Novel Soliton Molecules and Wave Interactions for A (3+1)-Dimensional Nonlinear Evolutionary Equation
}

Xiaoyan Tang ( $\nabla$ xytang@sist.ecnu.edu.cn )

East China Normal University

Chao Jie Cui

East China Normal University

Zu feng Liang

Hangzhou Normal University

Wei Ding

Shanghai Normal University

\section{Research Article}

Keywords: Soliton molecular, dromion, lump, ring soliton

Posted Date: March 12th, 2021

DOI: https://doi.org/10.21203/rs.3.rs-283620/v1

License: (c) (i) This work is licensed under a Creative Commons Attribution 4.0 International License.

Read Full License

Version of Record: A version of this preprint was published at Nonlinear Dynamics on July 23rd, 2021.

See the published version at https://doi.org/10.1007/s11071-021-06687-7. 


\title{
NOVEL SOLITON MOLECULES AND WAVE INTERACTIONS FOR A $(3+1)$-DIMENSIONAL NONLINEAR EVOLUTIONARY EQUATION
}

\author{
XIAO-YAN TANG ${ }^{1 *}$, CHAO-JIE CUI ${ }^{2}$, ZU-FENG LIANG ${ }^{3 *}$ AND WEI DING ${ }^{4}$ \\ ${ }^{1}$ School of Mathematical Sciences, Shanghai Key Laboratory of PMMP, East China Normal University, \\ Shanghai 200062, China \\ ${ }^{2}$ Shanghai Key Laboratory of Trustworthy Computing, East China Normal University, Shanghai 200062, \\ China \\ ${ }^{3}$ Department of Physics, Hangzhou Normal University, Hangzhou 310036, China \\ ${ }^{4}$ School of Computer Science and Technology, Shanghai Normal University, Shanghai 200234, China
}

\begin{abstract}
New wave excitations are revealed for a $(3+1)$-dimensional nonlinear evolution equation to enrich nonlinear wave patterns in nonlinear systems. Based on a new variable separation solution with two arbitrary variable separated functions obtained by means of the multilinear variable separation approach, localized excitations of $N$ dromions, $N \times M$ lump lattice and $N \times M$ ring soliton lattice are explored. Interestingly, it is observed that soliton molecules can be composed of diverse "atoms" such as the dromions, lumps and ring solitons, respectively. Elastic interactions between solitons and soliton molecules are graphically demonstrated.

Keywords: Soliton molecular; dromion; lump; ring soliton
\end{abstract}

\section{INTRODUCTION}

Nonlinear waves are universal in many fields such as fluids, plasma, optics, finance, and so on. Various nonlinear partial differential equations have been established to model abundant nonlinear waves. Meanwhile, many effective methods have been established to find exact solutions for different waves, such as the inverse scattering transform [1], the symmetry approaches [2], the Hirota's bilinear method [3], and various feasible transformation methods $[4,5]$ including the Bäcklund transformation and the Darboux transformation. Localized excitations in $(1+1)$ - and (2+1)-dimensional systems have been discussed in detail, ranging from one dimensional line-soliton, solitoff, kink and anti-kink to two dimensional exponentially decaying dromions, algebraically decaying lumps, time-periodic breathers, spatially periodic waves, rogue waves and multi-valued folded solitary waves. However, nonlinear waves in $(3+1)$-dimensions are less considered due to the difficulties in finding exact solutions for $(3+1)$-dimensional nonlinear systems.

It is known that the method of variable separation is one of the fundamental and effective methods for solving linear partial differential equations. Many efforts have been made to develop nonlinear versions of the linear variable separation method to find variable separation solutions for nonlinear partial differential equations. For instance, from the viewpoint of geometry, it was proposed that a solution of an evolution equation is separable if and only

Date: 14 December 2020.

* Corresponding author, email: xytang@sist.ecnu.edu.cn, liangzufeng@163.com. 
if it is a curve in the $(k+1)$-parameter space of characteristic fields [6]. The generalized conditional symmetry approach was used to study the separation of variables of quasilinear diffusion equations with nonlinear source, and a complete list of canonical forms was obtained for such equations admitting the functionally separable solutions [7]. By means of the symmetry constraints, a systematic variable separation approach has been established [8], although the independent variables of a reduced field have not been totally separated. As it was applicable only for integrable models with Lax pairs, the method was later extended to any models no matter whether they possess Lax pairs or not [9]. In order to realize the independent variables totally separated, the multilinear variable separation approach (MLVSA) and its generalizations $[10,11]$ have been established and then shown effective to obtain variable separation solutions of many nonlinear integrable systems. Recently, we applied the MLVSA to a (3+1)-dimensional Boiti-Leon-Manna-Pempinelli equation, yielding a new kind of variable separation solution with an arbitrary seed solution evolved in the potential fields [12], and thus, different from the previous results, the seed solution plays a role in the wave interactions with different background waves.

In this paper, we will focus on novel nonlinear localized wave excitations based on the variable separation solutions for the following $(3+1)$-dimensional nonlinear evolution equation

$$
3 u_{x z}-2 u_{t y}-u_{x x x y}+2 u u_{x y}+2\left(u_{x} \partial_{x}^{-1} u_{y}\right)_{x}=0 .
$$

This equation was first introduced in [13] to illustrate that it can be decomposed into three $(1+1)$-dimensional AKNS equations with the help of a direct way and the nonlinearization of a Lax pair. Therefore, algebraic-geometrical solutions expressed explicitly in terms of the Riemann theta functions were obtained. Later on, many types of solutions have been obtained, such as the $N$-soliton solutions, complexions, positons, negatons, rogue waves, breathers, and so on [14-18].

The paper is organized as follows. In section 2, a new variable separation solution with two arbitrary variable separated functions is obtained for Eq. (1), from which various nonlinear excitations and wave interactions on different wave backgrounds can be explored. In section 3 , three different restrictions are imposed on the arbitrary variable separated functions, and thus three types of nonlinear excitations including the dromions, lumps and ring solitons are obtained. Especially, when a pair of dromions, lumps or ring solitons have the same velocity, then they can be resonant to soliton molecules. Elastic interactions between a dromion and a dromion molecular, between a lump and a lump molecular, between two lump molecules, between a ring soliton and a ring soliton molecular are graphically illustrated, respectively. The last section is devoted to discussion and summary.

\section{VARIABlE SEPARATION SOLUTIONS}

It is easy to verify that through a Bäcklund transformation

$$
u=-3(\ln f)_{x x}+u_{0}+u_{1},
$$

where $u_{0} \equiv u_{0}(z, t), u_{1} \equiv u_{1}(x, t)$ are arbitrary seed solutions of (1), Eq. (1) can be converted into a general bilinear form

$$
\left(6 D_{y} D_{t}-9 D_{z} D_{x}+3 D_{x}^{3} D_{y}-6\left(u_{0}+u_{1}\right) D_{x} D_{y}+2 h_{1} x+2 h_{0}\right) f \cdot f=0,
$$


where $h_{0}$ and $h_{1}$ are two arbitrary functions of $(y, z, t)$, and the well known Hirota's bilinear operators $D_{i}(i=x, y, z, t)$ are defined by [4]

$$
\begin{aligned}
D_{x}^{m} D_{y}^{n} D_{z}^{r} D_{t}^{s} f \cdot f= & \left(\partial_{x}-\partial_{x^{\prime}}\right)^{m}\left(\partial_{y}-\partial_{y^{\prime}}\right)^{n}\left(\partial_{z}-\partial_{z^{\prime}}\right)^{r}\left(\partial_{t}-\partial_{t^{\prime}}\right)^{s} \\
& \left.f(x, y, z, t) \cdot f\left(x^{\prime}, y^{\prime}, z^{\prime}, t^{\prime}\right)\right|_{x^{\prime}=x, y^{\prime}=y, z^{\prime}=z, t^{\prime}=t} .
\end{aligned}
$$

Then assume the expansion function $f$ have a variable separated form of

$$
f=a_{0}+a_{1} r+a_{2} s+a_{3} r s
$$

where $a_{0}, a_{1}, a_{2}$ and $a_{3}$ are arbitrary constants, $r \equiv r(x, t)$ and $s \equiv s(y, z)$ are undetermined functions of the indicated variables. The substitution of (4) into (3) leads to

$$
\left(a_{0} a_{3}-a_{1} a_{2}\right)\left(s_{y} r_{x x x}+2 s_{y} r_{t}+3 s_{z} r_{x}-2\left(u_{0}+u_{1}\right) s_{y}\right)=0,
$$

with $h_{0}$ and $h_{1}$ fixed as zero. It is not difficult to reveal that requiring $a_{0} a_{3}-a_{1} a_{2} \neq 0$, the above equation (5) can be separated into two variable separated equations

$$
\begin{aligned}
& 2 r_{t}+r_{x x x}-2\left(u_{1}+h(t)\right) r_{x}=C, \\
& s_{z}-g^{\prime}(z) s_{y}=C,
\end{aligned}
$$

if $u_{0}$ is further assumed as $u_{0}=h(t)-3 g^{\prime}(z) / 2$, where $g$ and $h$ are two arbitrary functions of the indicated arguments, and the separation constant $C$ can be set zero without the loss of generality.

Solving Eqs. (6)-(7) gives

$$
u_{1}=\frac{2 r_{t}+r_{x x x}}{2 r_{x}}-h(t), \quad s=s(y+g(z)) .
$$

Consequently, we obtain a new multilinear variable separation solution (MLVSS) of (1) as

$$
u=\frac{2 r_{t}+r_{x x x}}{2 r_{x}}-\frac{3}{2} g^{\prime}(z)-\frac{3\left(a_{1}+a_{3} s\right) r_{x x}}{a_{0}+a_{1} r+a_{2} s+a_{3} r s}+\frac{3\left(a_{1}+a_{3} s\right)^{2} r_{x}^{2}}{\left(a_{0}+a_{1} r+a_{2} s+a_{3} r s\right)^{2}},
$$

where $g$ is an arbitrary function of $z, r$ is an arbitrary function of $(x, t)$, and $s$ is an arbitrary function of $(y+g)$.

It is remarkable that the arbitrary functions in the MLVSS (9) can be utilized to construct abundant nonlinear excitations. It is stressed that the first two terms in (9), coming from the seed solutions of (1), are responsible for different background waves, and thus one might be able to observe the interplay between the background waves and various excited waves determined by the last two terms in (9). Furthermore, it is worth emphasizing that for this particular model, the potentials of the field $u$, for instance,

$$
U \equiv u_{y}=\frac{6\left(a_{0} a_{3}-a_{1} a_{2}\right)\left(a_{3} s+a_{1}\right) s_{y} r_{x}^{2}}{\left(a_{3} r s+a_{1} r+a_{2} s+a_{0}\right)^{3}}-\frac{3\left(a_{0} a_{3}-a_{1} a_{2}\right) s_{y} r_{x x}}{\left(a_{3} r s+a_{1} r+a_{2} s+a_{0}\right)^{2}},
$$

cannot be expressed in the universal form applicable for a number of integrable systems [10-12]. 


\section{NONLINEAR EXCITATIONS AND THEIR INTERACTIONS}

As indicated above, various wave interactions can be explored for the sake of the arbitrary functions in the MLVSS (9). Recently, soliton molecules have attracted much attention for their continuous discoveries in nonlinear optical experiments [19-22]. As a special type of nonlinear excitations, soliton molecules have also been demonstrated in other physical systems, such as in dipolar Bose-Einstein condensates [23]. Soliton molecules come along from some fundamental solitons acting as "atoms" [24]. It has been mathematically revealed that a soliton molecule can be generated from an exact two-soliton solution [25]. A new mechanism, the velocity resonant, has been proposed to find soliton molecules $[26,27]$. Hinted by this scheme, we have found interesting dromion molecules in a dromion lattice [12]. It is well known that dromions, lumps, compactons, foldons and many other localized excitations can be explained by a MLVSS. In this section, we will just focus on the localized waves the types of dromion, lump and ring soliton, construct soliton molecules with two "atoms" of dromions, lumps or ring solitons, and finally study their interactions.

3.1. Dromions, dromion molecules and their interactions. To explore dromion excitations from the MLVSS, we restrict the variable separated functions $r$ and $s$ as

$$
r=c+\sum_{i=1}^{N} \exp \left[\omega_{i}-\left(k_{i} x+v_{i} t+b_{i}\right)^{2}\right], \quad s=C+\exp \left[\Omega-\left(K_{1} y+\operatorname{sech}\left(L_{1} z\right)\right)^{2}\right],
$$

where $k_{i}, v_{i}, b_{i}, \omega_{i}, K_{1}, L_{1}, c, C$ and $\Omega$ are constants. In order to make it easy to graphically display wave interactions described by the potential field $u_{y}(10)$, we simply take $z=5$. In this reduced space-time $\{x, y, t\}$, the potential quantity $U\left(\equiv u_{y}\right)$ given by (10) with (11) can describe various dromion behaviours. In addition, the arbitrary constants $v_{i}$ and $\omega_{i}$ determine the velocities and shapes of the dromions, respectively.

Case 1. Dromion-dromion interaction. If we set $a_{0}=2, a_{3}=0, a_{1}=a_{2}=1$ and fix the parameters in (11) as $N=2, c=\omega_{1}=v_{1}=-v_{2}=3, K_{1}=L_{1}=k_{1}=k_{2}=1, \omega_{2}=$ $2, C=\Omega=0$ and $b_{1}=-b_{2}=5$, two dromions are obtained as shown in Fig. 1, which travel in the opposite directions along the $x$ axis, and interact at time $t=-5 / 3$. It is clear that the collision between these two dromions is completely elastic, because the dromions preserve their shapes and velocities during the interaction.

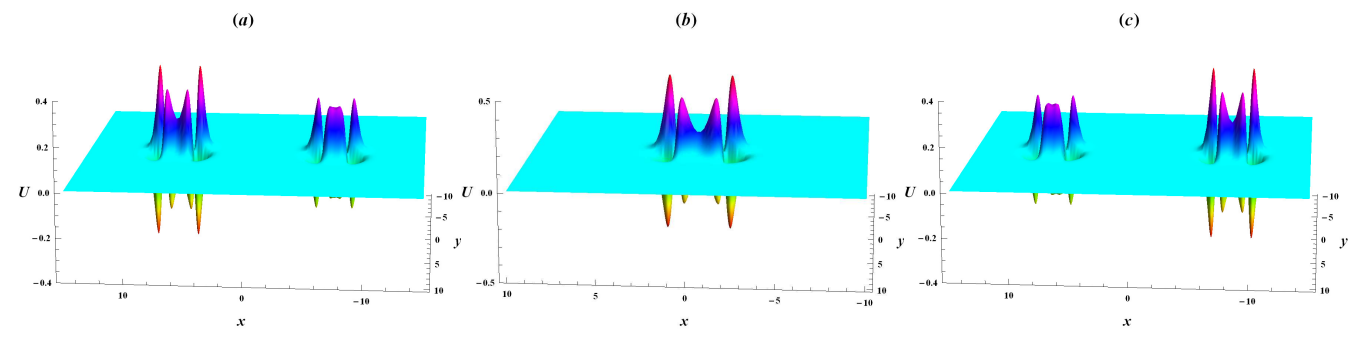

Figure 1. Elastic interactions between two dromions with the opposite velocities at times $(a) t=-4,(b) t=-5 / 3$, and $(c) t=1$, respectively.

Case 2. Dromion molecules. It is known that two dromions can form a dromion molecular when they have a same velocity, inherited from the velocity resonant mechanism. Hence, if 
the velocities of two dromions in Fig. 1 are replaced by $v_{1}=v_{2}=3$, then two dromions are bounded as a dromion molecular and move steadily along the $x$ axis, as displayed in Fig. 2. It is clearly displayed that this dromion molecular is asymmetric because two dromion "atoms" possess different three dimensional structures. As is mentioned, the shapes of dromions are determined by the parameters $\omega_{i}$. Therefore, if fixing $\omega_{1}=\omega_{2}=3$, then a symmetric dromion molecular can be generated as exhibited in Fig. 3 .
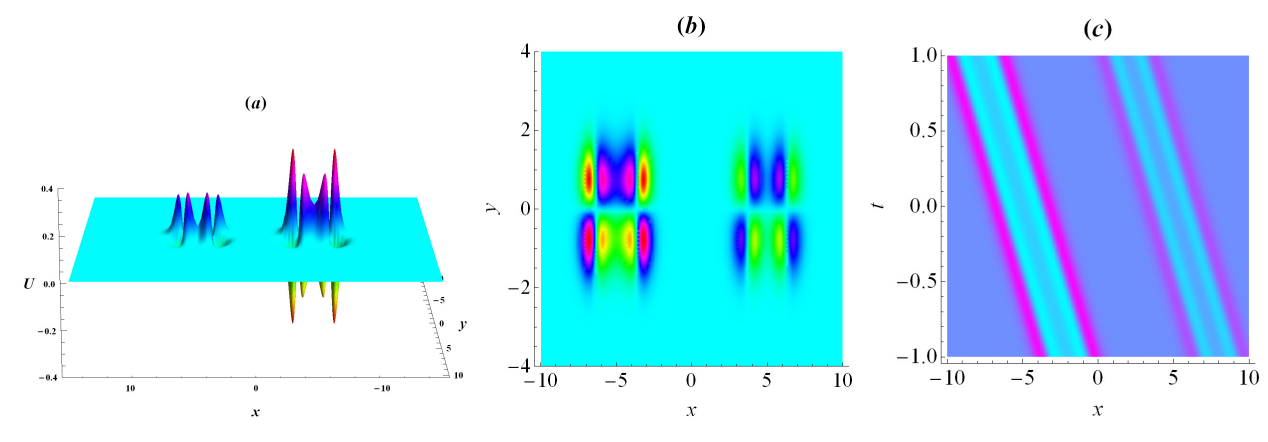

Figure 2. An asymmetrical dromion molecular (a) 3D plot at time $t=0$; (b) density plot at time $t=0$, and (c) time evolution from $t=-1$ to $t=1$.
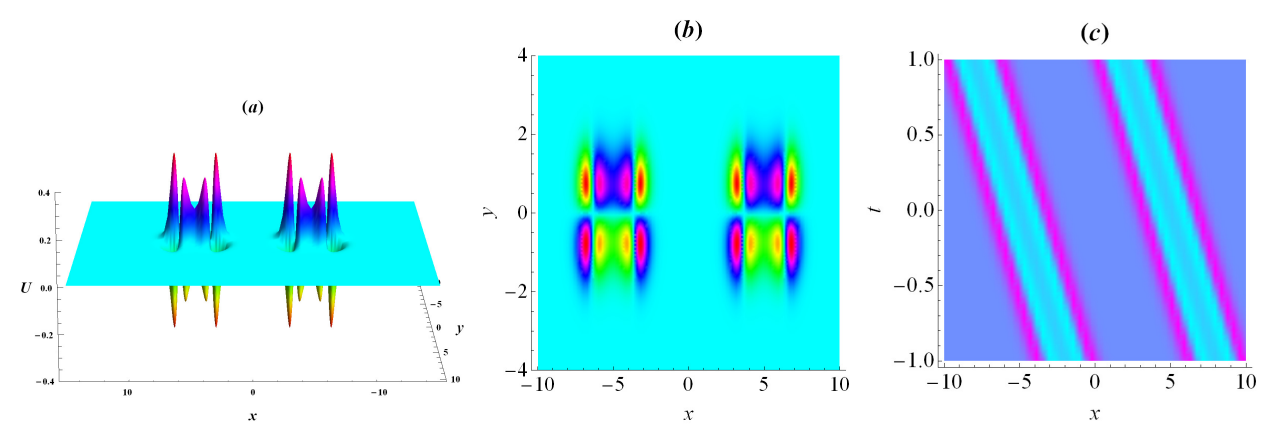

Figure 3. A symmetrical dromion molecular (a) 3D plot at time $t=0$; (b) densityplot at time $t=0$, and (c) time evolution from $t=-1$ to $t=1$.

Case 3. Dromion-dromion molecular interaction. It is noted that the restriction (11) is a building block for $N$ dromions, and thus various molecules constituted by different numbers of either symmetric or asymmetric dromions can be produced. Besides, it is also feasible to investigate nonlinear interactions between dromions and dromion molecules. Here, we just consider the simplest case with $N=3$ for a dromion and a symmetric dromion molecular. The related parameters are taken as $c=\omega_{3}=v_{1}=-v_{2}=-v_{3}=3, K_{1}=L_{1}=k_{1}=k_{2}=$ $k_{3}=b_{3}=1, \omega_{1}=\omega_{2}=2, C=\Omega=0$ and $b_{1}=-b_{2}=5$. In this particular setting, an independent dromion interacts with the dromion "atoms" of a symmetric dromion molecular one by one at time $t=-5 / 3$ and $t=-1 / 3$, respectively. It is also graphically demonstrated in Fig. 4 that after the collision, the dromion and the dromion molecular remain their shapes and velocities, therefore, they have experienced a completely elastic interaction. 


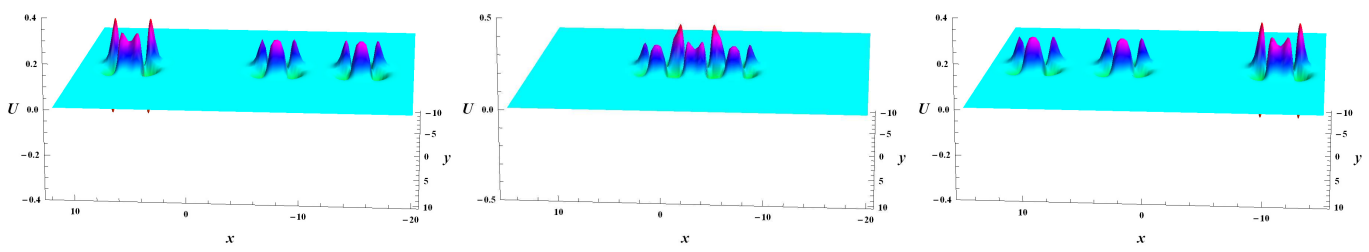

FiguRE 4. An elastic interaction between a dromion and a symmetrical dromion molecular at times $(a) t=-4,(b) t=-1$, and $(c) t=2$, respectively.

3.2. Lumps, lump molecules and their interactions. Now we suppose the variable separated functions $r$ and $s$ in the form of

$$
r=c+\sum_{i=1}^{N} \frac{h_{i}}{\omega_{i}-\left(k_{i} x+v_{i} t+b_{i}\right)^{2}}, \quad s=C+\sum_{i=1}^{M} \frac{H_{i}}{\Omega_{i}-\left(K_{i} y+V_{i} z+B_{i}\right)^{2}},
$$

where $c, C, k_{i}, v_{i}, b_{i}, h_{i}, \omega_{i}, K_{i}, V_{i}, B_{i}, H_{i}$ and $\Omega_{i}$ are all arbitrary constants. With this assumption, the potential quantity $U$ makes it possible to observe interesting nonlinear wave patterns of lumps and lump molecules. It is not difficult to verify that the potential field $U$ given by Eq. (10) with (12) describes an $N \times M$ lump lattice, and then by adjusting the velocities and shapes of its lumps, different symmetric and asymmetric lump molecules could be generated which possess different numbers of symmetric or asymmetric lumps. It is remarkable that the rule of $\bigoplus$ defined in Ref. [12] is also applicable in this case. Hereafter we take $z=a_{3}=2$ and $a_{0}=a_{1}=a_{2}=1$ for simplicity.

Case 1. Lump molecules with $N=M=2$. In this case, we have a lump lattice constructed by $2 \times 2$ lumps, namely, four lumps are situated in two rows and two columns. Take other parameters as $c=v_{1}=k_{1}=k_{2}=h_{1}=K_{1}=K_{2}=V_{1}=V_{2}=1, h_{2}=v_{2}=3, b_{1}=-b_{2}=$ $\omega_{1}=\omega_{2}=C=H_{1}=H_{2}=5,-B_{1}=B_{2}=8$ and $\Omega_{1}=\Omega_{2}=1 / 5$. Consequently, two lumps in each column are identical and bounded through their same velocities, and hence two symmetric lump molecules appear, as shown in Fig. 5. It is observed that the interaction between two symmetric lump molecules is completely elastic and they fully interact each other at time $t=2.5$.
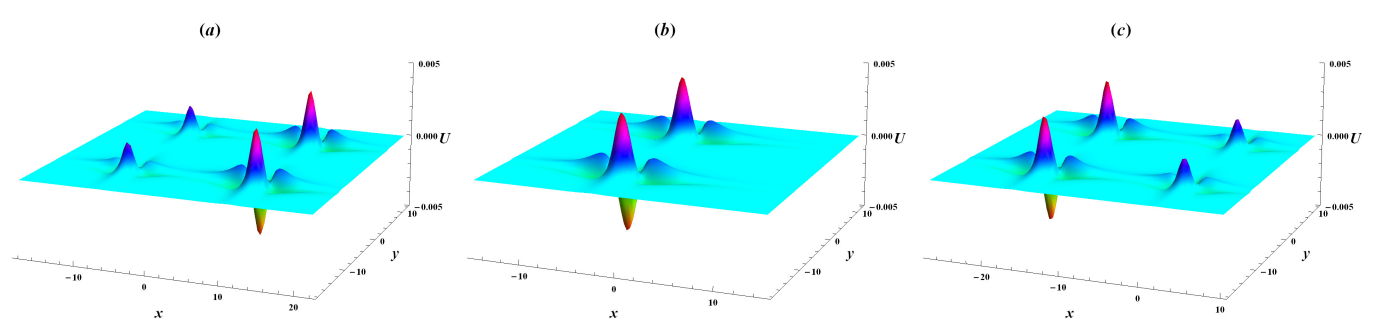

Figure 5. An elastic interaction betwen two symmetric lump molecules at times $(a) t=-2,(b) t=2.5$, and $(c) t=7$, respectively. 
If the values of $h_{1}$ and $\Omega_{1}$ are replaced by $h_{1}=3$ and $\Omega_{1}=1$, respectively, then two asymmetric lump molecules come in being, and they move in the opposite directions along the $x$-axis, and also interact elastically and fully at time $t=2.5$, as exhibited in Fig. 6 .

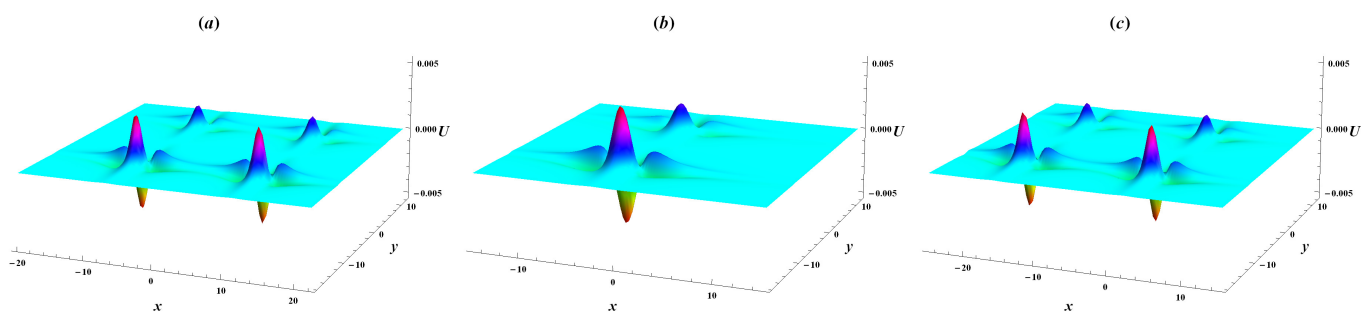

FiguRE 6. An elastic interaction between two asymmetrical lump molecules at times $(a) t=-2,(b) t=2.5$, and $(c) t=7$, respectively.

Case 2. Lump-lump molecular interaction with $N=3$ and $M=1$. Here a simplest interaction is considered between a lump and a symmetric lump molecular. More complicated interactions can be explored by introducing more lumps and lump molecules. Fig. 7 displays an elastic interaction between a lump and a symmetric lump molecular under the parameters $c=h_{1}=\omega_{3}=v_{2}=v_{3}=b_{3}=3, K_{1}=V_{1}=k_{1}=k_{2}=k_{3}=h_{2}=h_{3}=-v_{1}=1, C=\omega_{1}=$ $\omega_{2}=\omega_{3}=H_{1}=b_{1}=-b_{2}=5, B_{1}=-8$ and $\Omega=1 / 5$. In this case, the lump interacts with two lump "atoms" of the symmetric lump molecular one by one at time $t=-1$ and $t=2.5$, respectively.
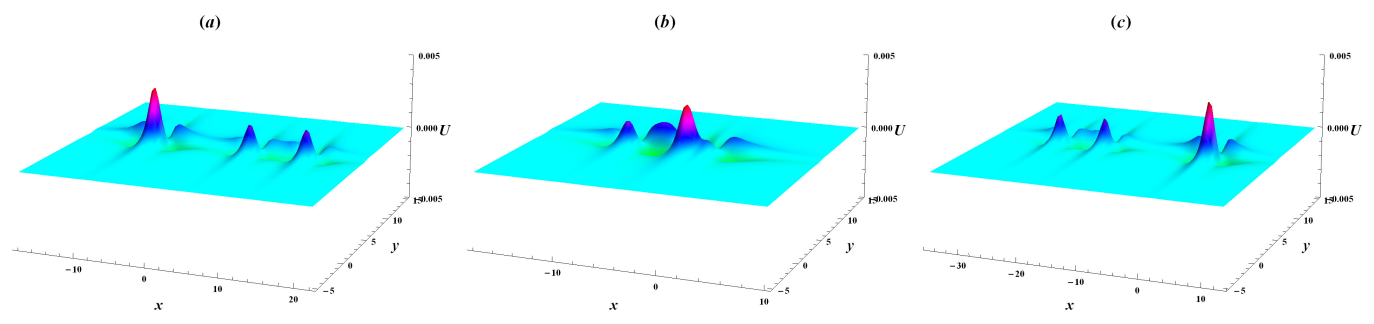

FigurE 7. An elastic interaction between a lump and a symmetrical lump molecular at times $(a) t=-3,(b) t=2$, and $(c) t=7$, respectively.

3.3. Ring solitons, ring soliton molecules and their interactions. In some special situations, one can have a solution whose value is nonzero only on a closed curve while decays exponentially away from the curve. This typical wave pattern, named a ring soliton, has been found its existence in various $(2+1)$-dimensional integrable systems [10]. Therefore, it is possible to search for similar ring solitons in a reduced $(3+1)$-dimensional setting. After some trails, we discover the following requirement for the variable separated functions $r$ and $s$

$$
r=c+\sum_{i=1}^{N} \exp \left[\omega_{i}-\left(k_{i} x+v_{i} t+b_{i}\right)^{2}\right], \quad s=C+\sum_{i=1}^{M} \cosh \left[\Omega_{i}+\left(K_{i} y+V_{i} z+B_{i}\right)^{2}\right],
$$


with some arbitrary constants $c, C, k_{i}, v_{i}, b_{i}, h_{i}, \omega_{i}, K_{i}, V_{i}, B_{i}, H_{i}$ and $\Omega_{i}$, can be used to build up ring solitons for the potential field $U$ with $a_{0}=2, a_{3}=0, a_{1}=a_{2}=1$ in the reduced space-time $\{x, y, t\}$ by setting $z=0$. It is emphasized that the ratios of $v_{i} / k_{i}$ and $V_{i} / K_{i}$ gives the velocities of the ring solitons, $b_{i}$ and $B_{i}$ decide the center of the ring solitons, and the radius (and thus the amplitudes) of the ring solitons are determined by $\omega_{i}$ and $\Omega_{i}$.

Case 1. Ring solitons. First to take $N=M=1$ to show a ring soliton in detail with the parameters fixed as $c=C=-K_{1}=k_{1}=V_{1}=-v_{1}=\Omega_{1}=1, \omega_{1}=10$ and $B_{1}=b_{1}=5$. In this case, a novel crossed saddle-type ring soliton is constructed for the potential field $U$ as shown in Fig. 8 (a). It is not difficult to verify that the potential field $U$ decays exponentially to zero away from the cirle $(x+5)^{2}+(y+5)^{2}=9$, which means the ring soliton has its center at $(-5,-5)$ and a radis 3 , as is clearly demonstrated in Fig. 8 (b).

It is pointed out above that it is also possible to explore the interplay between the excited wave and the wave background. Here it is simply show that corresponding to the potential field $U$ in Fig. 8, the original field $u$ given by (9) also captures a salient feature of a ring soliton while situated on a parabolic like wave background, as exhibited in Fig. 8 (c).
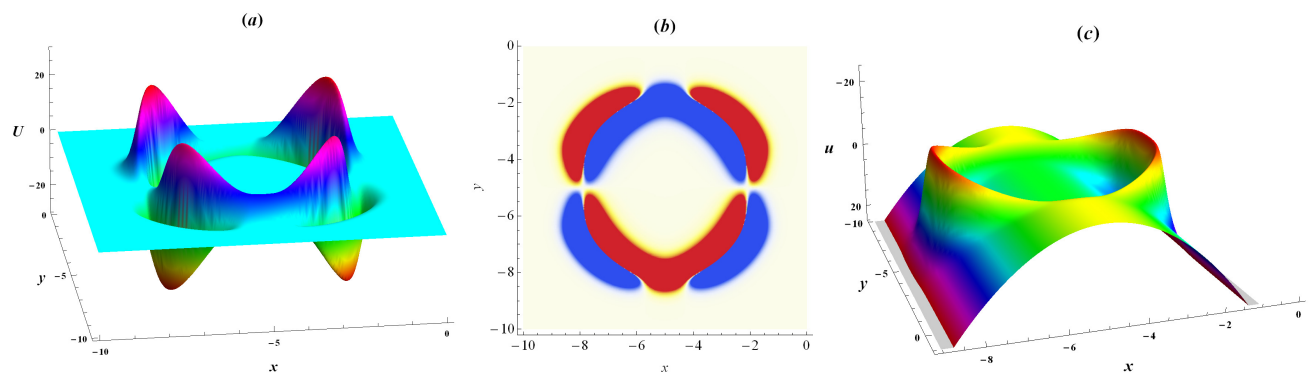

Figure 8. A novel ring-type soliton at time $t=0$ for (a) the potential field $U$, (b) densityplot of $U$ in (a), and (c) the original field $u$.

Case 2. Ring soliton-ring soliton molecular interactions. Setting $N=3$ and $M=1$, we may have three ring solitons. Under the parameters $c=C=k_{1}=k_{2}=k_{3}=K_{1}=V_{1}=$ $-v_{1}=-v_{2}=v_{3}=\Omega_{1}=1, \omega_{1}=\omega_{2}=10, \omega_{3}=15$ and $b_{1}=-b_{2}=-b_{3}=B_{1}=5$, the potential field $U$ possesses a three-ring-soliton excitation, among which two small ring solitons share the same structure and velocity and thus bounded as a symmetric ring soliton molecular, and it interacts elastically with a big ring soliton, as shown in Fig. 9.

It is observed that the big ring soliton interacts with the ring soliton "atoms" of the symmetric ring soliton molecular one by one at time $t=0$ and 5 , respectively. After the interactions, they all restore their structures and velocities, which demonstrates it is really a completely elastic collision. In addition, two "atoms" of the symmetric ring soliton molecular have the same radius 3 and their center are located at $(-5, t-5)$ and $(-5, t+5)$, respectively, while the big ring soliton with the radius $\sqrt{14}$ has its center at $(-5,5-t)$.

If $\omega_{2}$ is set as 15 instead of 10 , then one finds an asymmetric ring soliton molecular whose "atoms" located at $(-5, t-5),(-5, t+5)$ with the radius 3 and $\sqrt{14}$, respectively. Similarly, the asymmetric ring soliton molecular interacts elastically with the ring soliton, as shown in the upper line in Fig. 10 for the potential field $U$. Fig. 10(d)-(f) display the related nonlinear excitations for the original field $u$ corresponding to (a)-(c), accordingly. 
(a)

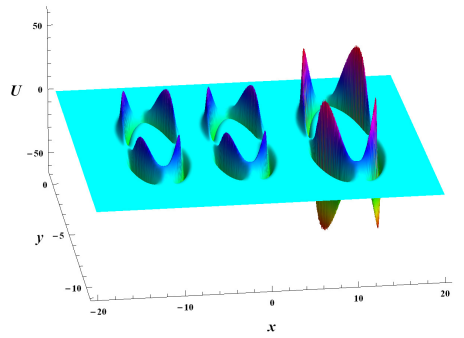

(b)

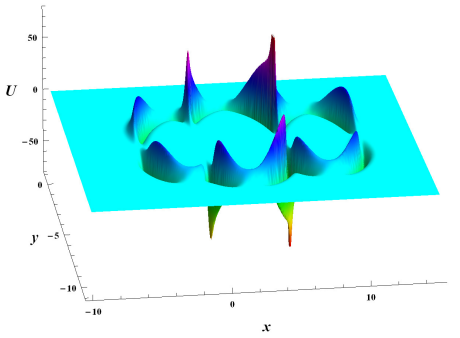

(c)

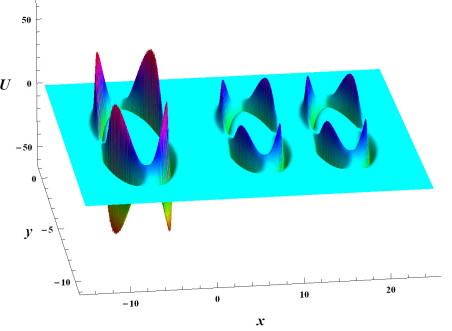

FiguRE 9. An elastic interaction betwee a symmetrical ring soliton molecular and a ring soliton at time $(a) t=-6,(b) t=3$, and $(c) t=12$, respectively.
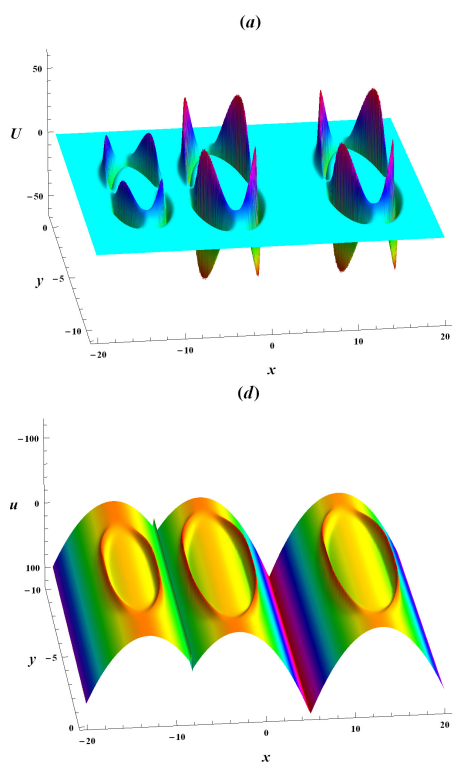

(a)
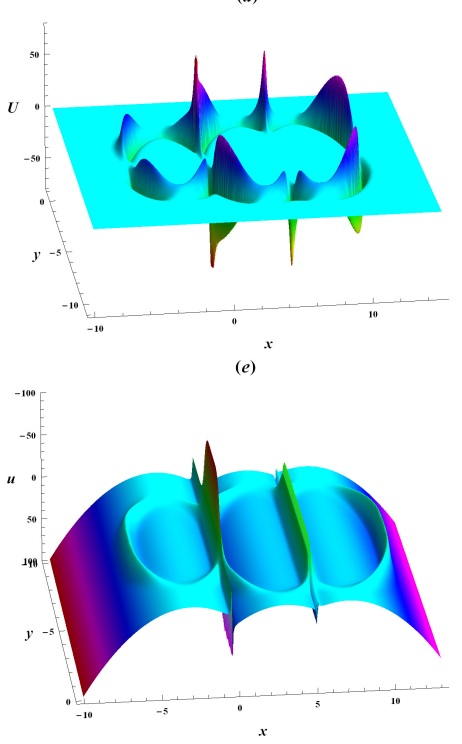

(a)
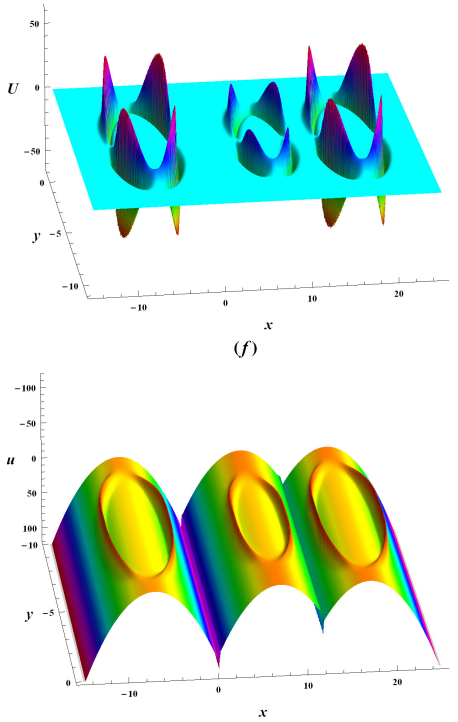

FigURE 10. An ealstic interaction between an asymmetric ring soliton molecular and a ring soliton at time $(a, d) t=-8,(b, e) t=2$, and $(c, f) t=12$ for the potential field $U$ (upper line), and the original field $u$ (lower line), respectively.

\section{Summary AND DisCUSSiON}

In summary, with the help of the multi-linear variable separation approach, we have obtained a new variable separation solution for a $(3+1)$-dimensional nonlinear evolution equation. Three types of selections have been chosen for the arbitrary variable separated functions to describe three different excitations of $N$ dromions, $N \times M$ lump lattice and $N \times M$ ring soliton lattice, respectively, whose sizes, velocities and locations are determined by some crucial parameters. Following the velocity resonant mechanism, soliton molecules can be easily produced by adjusting some dromions, lumps or ring solitons to have the same velocity.

In detail, soliton molecules with two "atoms" are discussed, including the dromion molecular, lump molecular and ring soliton molecular. It is graphically demonstrated that these soliton molecules can be asymmetric or symmetric, depending on their "atom" solitons are 
of the same or not. It is also found that interactions between the dromion molecular and the dromion, between the lump molecular and the lump, between two lump molecules either symmetric or asymmetric, between the ring soliton molecular and the ring soliton are completely elastic. In addition, it is revealed that the original field shares a similar nonlinear excitation for the potential field except for an extra parabolic-like wave background.

The findings in this work enrich the studies of soliton molecules. More abundant soliton molecules can be explored in this mathematical setting, however, the physical background of these soliton melecules is still not clear and also the mechanism of their appearance, which deserves further investigations.

\section{ACKNOWLEDGEMENT}

The authors acknowledge the financial support by the National Natural Science Foundation of China (No.11675055) and the Science and Technology Commission of Shanghai Municipality (No. 18dz2271000).

\section{COMPLIANCE WITH ETHICAL STANDARDS}

\section{CONFLICT OF INTEREST}

The authors declare that they have no conflict of interest.

\section{REFERENCES}

[1] C.S. Gardner, J.M. Greene, M.D. Kruskal, R.M. Miura, Method for solving the Korteweg-de Vries equation, Phys. Rev. Lett. 19 (1967) 1095-1097.

[2] P. J. Olver, Application of Lie Group to Differential Equations, Springer, New York, 1986.

[3] A.M. Wazwaz, Multiple-soliton solutions for the KP equation by Hirota's bilinear method and by the tanh-coth method, Appl. Math. Comput. 190 (2007) 633-640.

[4] R. Hirota, A New Form of Bäcklund Transformations and Its Relation to the Inverse Scattering Problem, Prog. Theor. Phys. 52 (1974) 1498-1512.

[5] V.B. Matveev, M.A. Salle, Darboux Transformations and Solitons, Springer, Berlin, 1991.

[6] P.W. Doyle, Separation of variables for scalar evolution equations in one space dimension, J. Phys. A: Math. Gen. 29 (1996) 7581-7595.

[7] C.Z. Qu, S.L. Zhang and R.C. Liu, Separation of variables and exact solutions to quasilinear diffusion equations with nonlinear source, Physica D 144 (2000) 97-123.

[8] Y.B. Zeng, A family of separable integrable Hamiltonian systems and their classical dynamical r-matrix Poisson structures, Inverse Problems 12 (1996) 797-809.

[9] S.Y. Lou and L.L. Chen, Formal variable separation approach for nonintegrable models, J. Math. Phys. 40(1999) 6491-6500.

[10] X.Y. Tang, S.Y. Lou, Y. Zhang, Localized excitations in (2+1)-dimendsional systems, Phys. Rev. E 66 (2002) 046601. 
[11] X.Y. Tang, S.Y. Lou, Extended multilinear variable separation approach and multivalued localized excitations for some (2+1)-dimensional integrable systems, J. Math. Phys. 44 (2003) 4000-4025.

[12] C.J. Cui, X.Y. Tang and Y.J. Cui, New variable separation solutions and wave interactions for the $(3+1)$-dimensional Boiti-Leon-Manna-Pempinelli equation, Appl. Math. Lett. 102 (2020) 106109.

[13] X.G. Geng, Algebraic-geometrical solutions of some multidimensional nonlinear evolution equations, J. Phys. A. Math. Gen. 36 (2003) 2289-2304.

[14] Jingjing Xie, Xiao Yang, Rogue waves, breather waves and solitary waves for a $(3+1)$ dimensional nonlinear evolution equation, Appl. Math. Lett. 97 (2019) 6-13.

[15] Q.L. Zha, Z.B. Li, Darboux transformation and various solutions for a nonlinear evolution equation in (3+1)-dimensions, Modern Phys. Lett. B 22 (2008) 2945-2966.

[16] Q.L. Zha, Z.B. Li, Positon, negaton, soliton and complexiton solutions to a fourdimensional evolution equation, Moderm Phys. Lett. B 23 (2009) 2971-2991.

[17] X.G. Geng, Y.L. Ma, N-soliton solution and its wronskian form of a (3+1)-dimensional nonlinear evolution equation, Phys. Lett. A 369 (2007) 285-289.

[18] J.P. Wu, A Bäcklund transformation and explicit solutions of a (3+1)-dimensional soliton equation, Chin. Phys. Lett. 25 (2008) 4192-4194.

[19] M. Stratmann, T. Pagel and F. Mitschke, Experimental observation of temporal soliton molecules, Phys. Rev. Lett. 95 (2005) 143902.

[20] G. Herink, F. Kurtz, B. Jalali, D.R. Solli and C. Ropers, Real-time spectral interferometry probes the internal dynamics of femtosecond soliton molecules, Science 356 (2017) $50-54$.

[21] X.M. Liu, X.K. Yao and Y.D. Cui, Real-time observation of the buildup of soliton molecules, Phys. Rev. Lett. 121 (2018) 023905.

[22] R. Xia, Y.Y. Luo, P.P. Shum, W.J. Ni, Y.S. Liu, H.Q. Lam, Q.Z. Sun, X.H. Tang and L.M. Zhao, Experimental observation of shaking soliton molecules in a dispersionmanaged fiber laser, Optics Lett. 45 (2020) 1551-1554.

[23] B.B. Baizakov, S.M. Al-Marzoug, U. Al. Khawaja and H. Bahlouli, Weakly bound solitons and two-soliton molecules in dipolar Bose-Einstein condensates, J. Phys. B: At. Mol. Opt. Phys. 52 (2019) 095301.

[24] L.C. Crasovan, Y.V. Kartashov, D. Mihalache, L. Torner, Y.S. Kivshar, V.M. PérezGarcía, Soliton "molecules": robust clusters of spatiotemperal optical solitons, Phys. Rev. E 67 (2003) 046610.

[25] U.Al. Khawaja, Stability and dynamics of two-soliton molecules, Phys. Rev. E 81 (2010) 056603.

[26] S.Y. Lou, Soliton molecules and asymmetric solitons in fluid systems via velocity resonance, (2019) arXiv:1909.03399 [nlin.SI].

[27] S.Y. Lou, Soliton molecules and asymmetric solitons in three fifth order systems via velocity resonance, J. Phys. Commun. 4 (2020) 041002. 
Figures

(a)

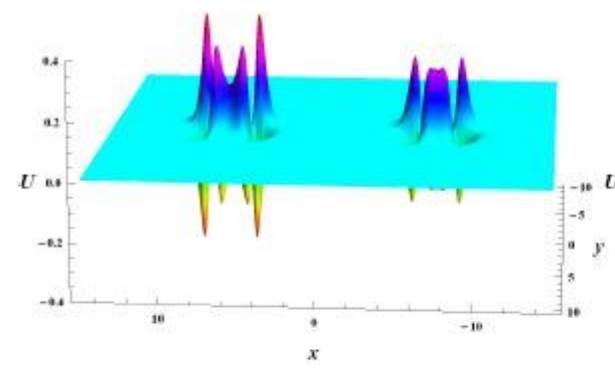

(b)

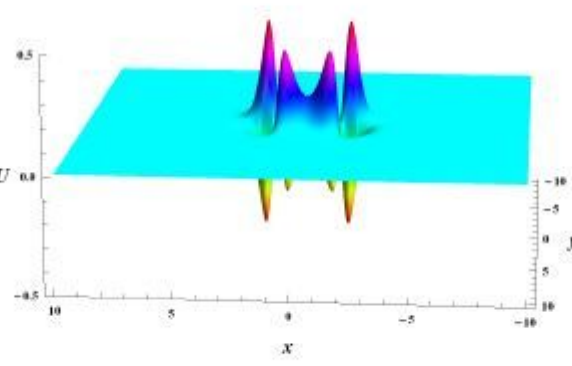

(c)

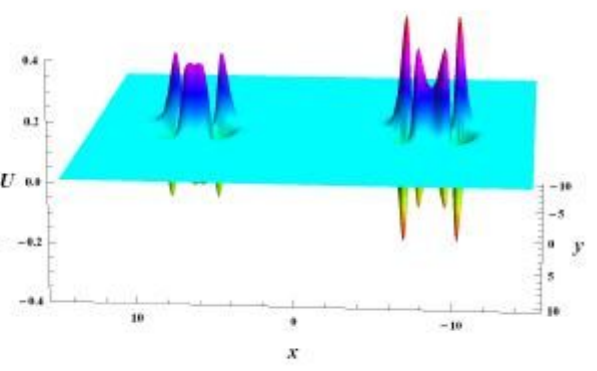

Figure 1

Elastic interactions between two dromions with the opposite velocities at times (a) $t=-4$, (b) $t=-5=3$, and (c) $t=1$, respectively.

(b)

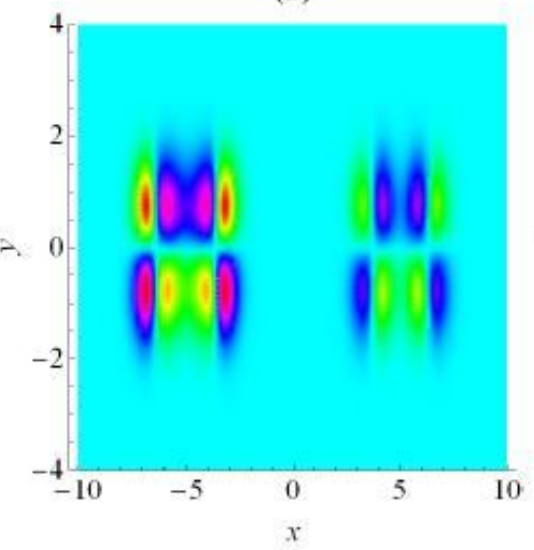

(c)

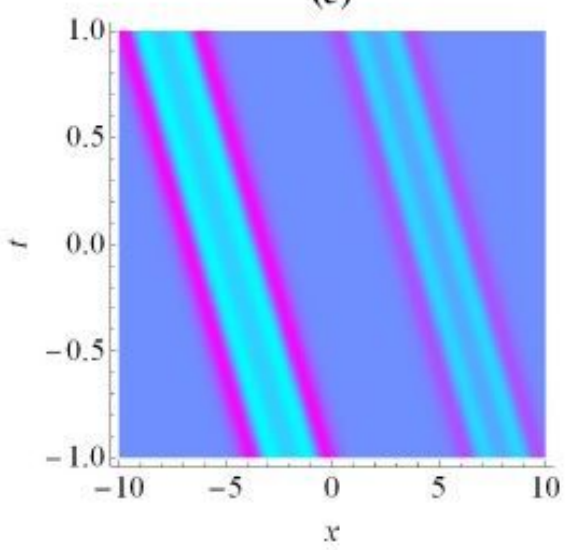

Figure 2

An asymmetrical dromion molecular (a) 3D plot at time $t=0$; (b) density plot at time $t=0$, and (c) time evolution from $t=-1$ to $t=1$. 
(b)

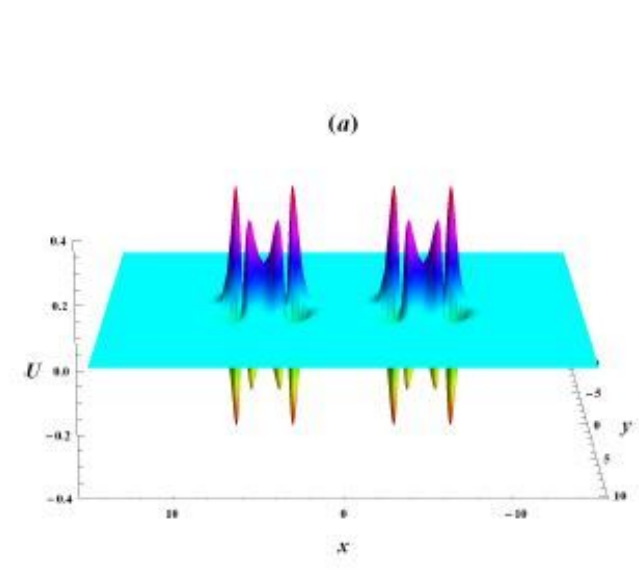

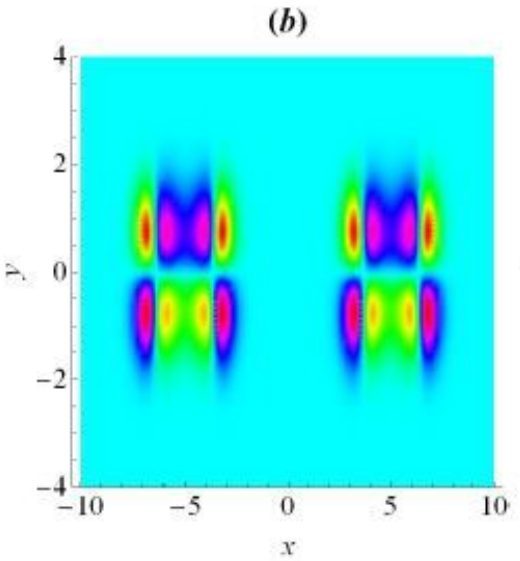

(c)

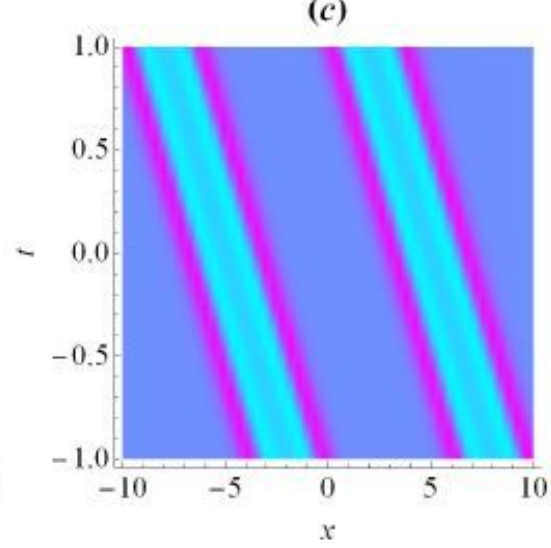

Figure 3

A symmetrical dromion molecular (a) 3D plot at time $t=0$; (b) densityplot at time $t=0$, and (c) time evolution from $t=-1$ to $t=1$.

(a)

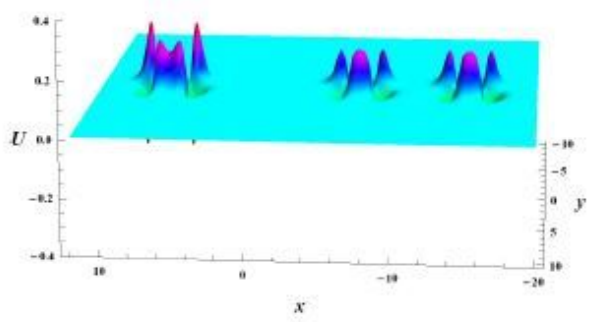

(b)

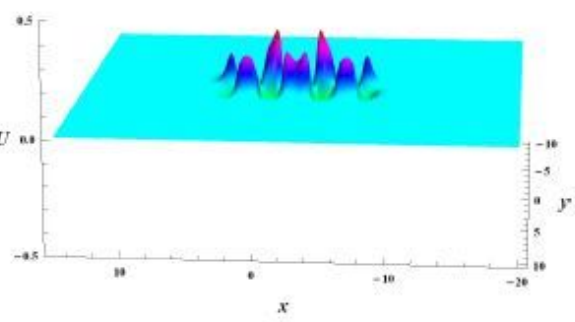

(c)

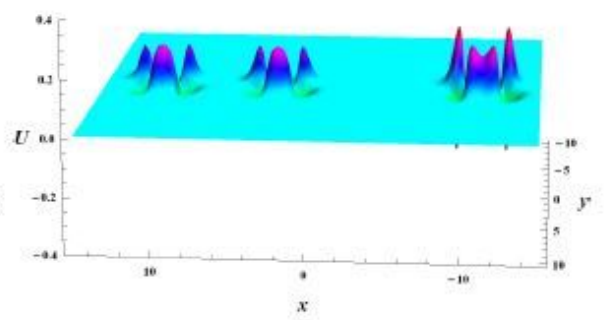

Figure 4

An elastic interaction between a dromion and a symmetrical dromion molecular at times $(a) t=-4$, (b) $t=$ -1 , and (c) $t=2$, respectively.

(a)

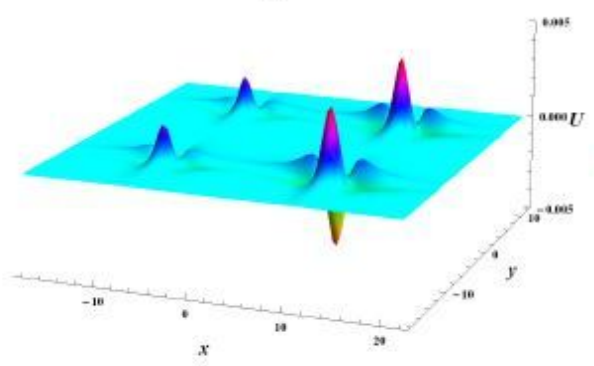

(b)

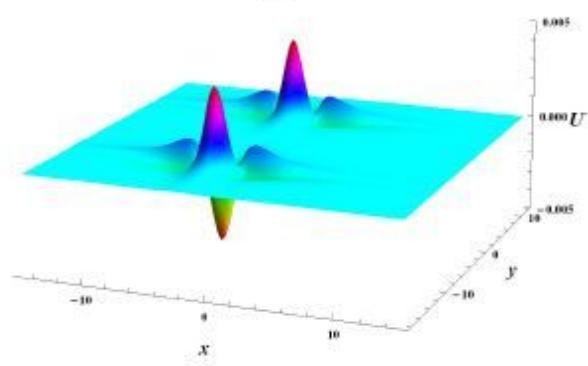

(c)

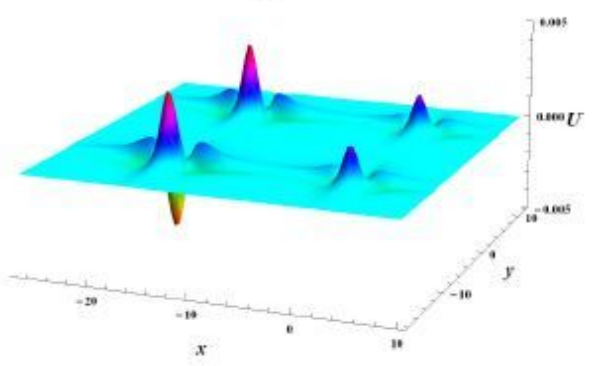

Figure 5 
An elastic interaction betwen two symmetric lump molecules at times (a) $t=-2$, (b) $t=2: 5$, and (c) $t=7$, respectively.

(a)

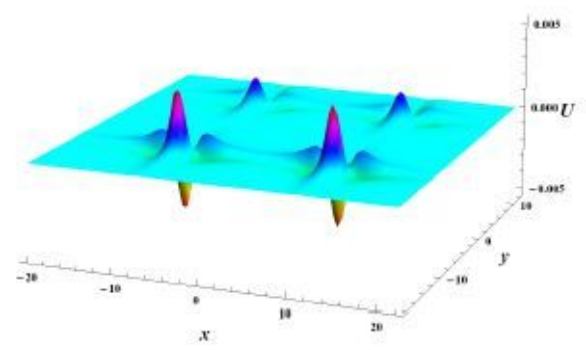

(b)

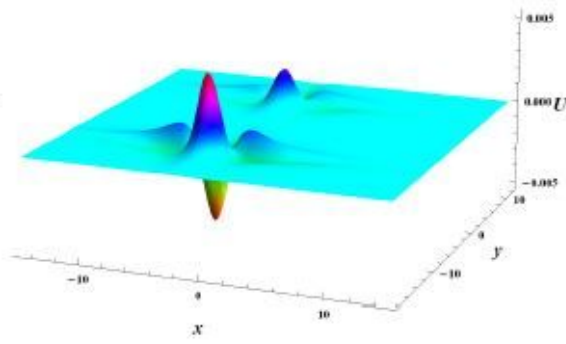

(c)

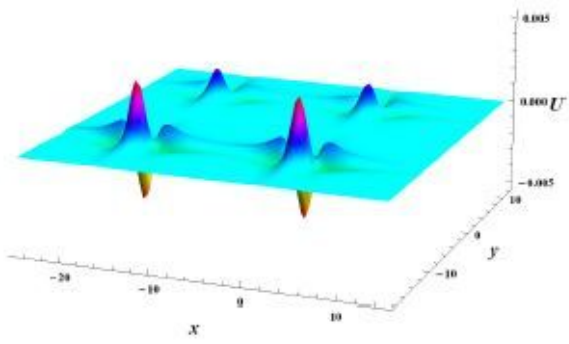

Figure 6

An elastic interaction between two asymmetrical lump molecules at times (a) $t=-2$, (b) $t=2: 5$, and (c) $t=$ 7 , respectively.

(a)

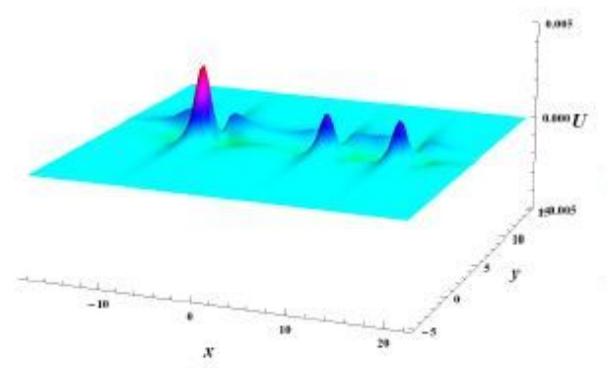

(b)

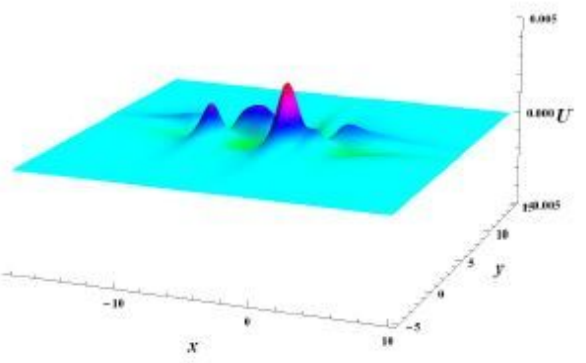

(c)

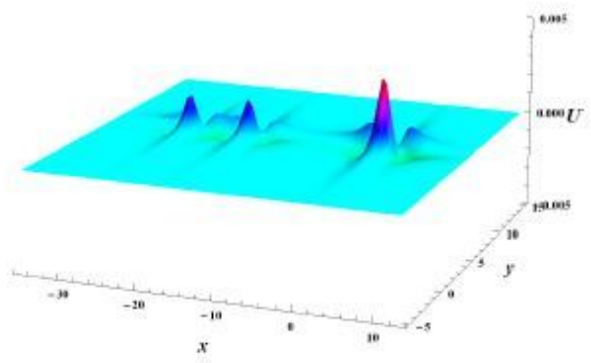

Figure 7

An elastic interaction between a lump and a symmetrical lump molecular at times (a) $t=-3$, (b) $t=2$, and (c) $t=7$, respectively.

(a)

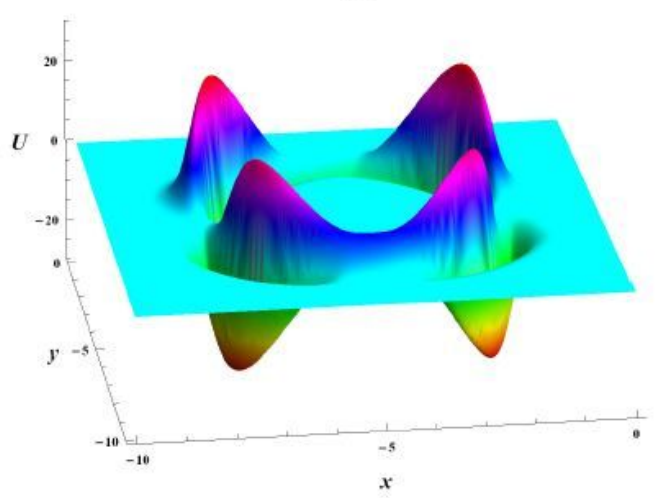

(b)

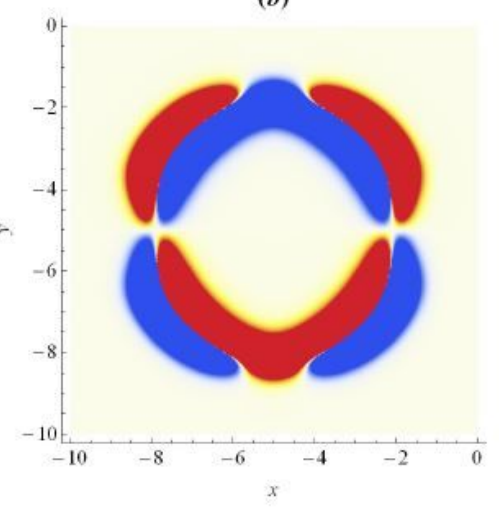

(c)

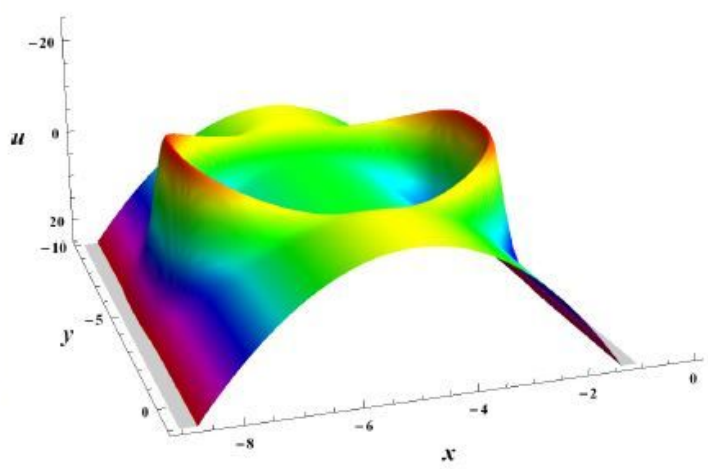

Figure 8 
A novel ring-type soliton at time $t=0$ for $(a)$ the potential eld $U,(b)$ densityplot of $U$ in (a), and (c) the original eld $u$.

(a)

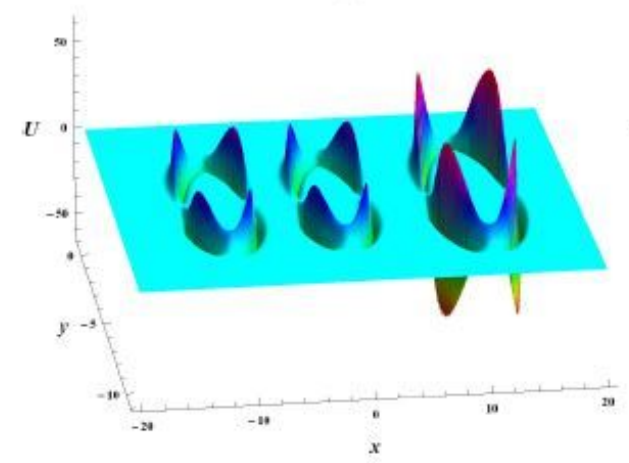

(b)

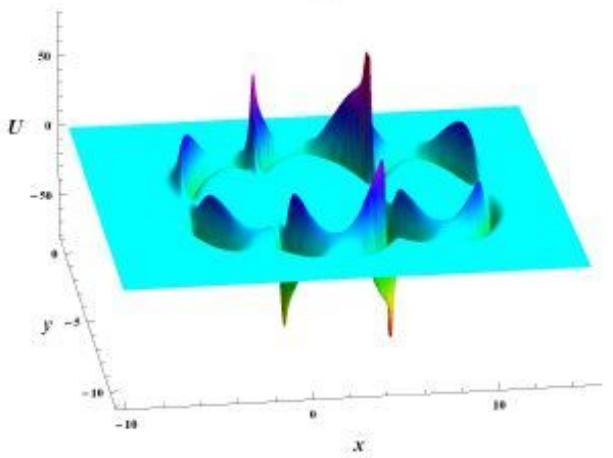

(c)

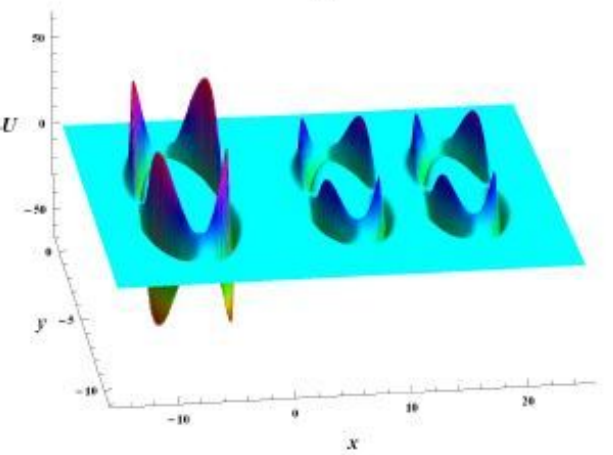

\section{Figure 9}

An elastic interaction betwee a symmetrical ring soliton molecular and a ring soliton at time $(a) t=-6,(b) t$ $=3$, and $(\mathrm{c}) \mathrm{t}=12$, respectively.

(a)
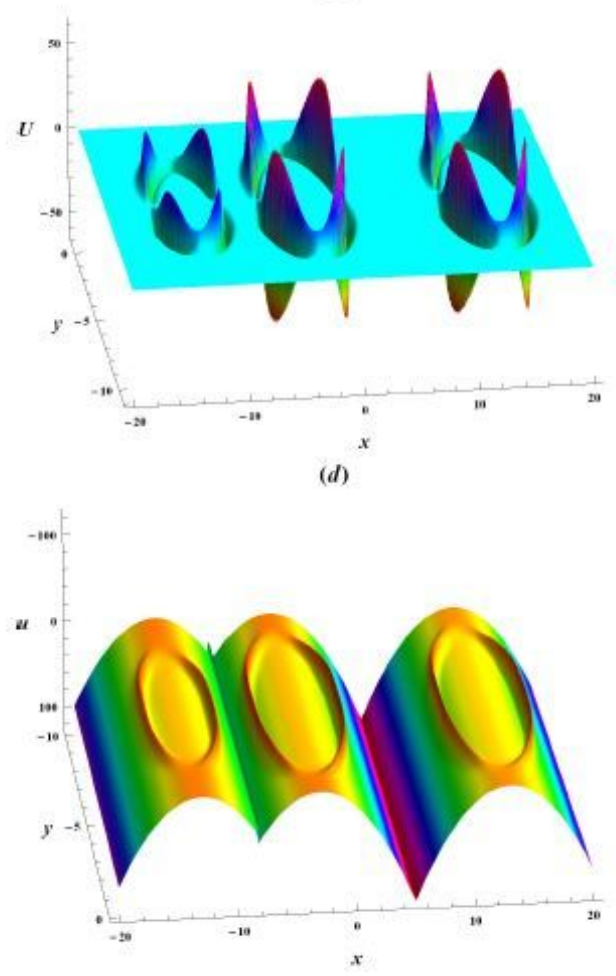

(a)

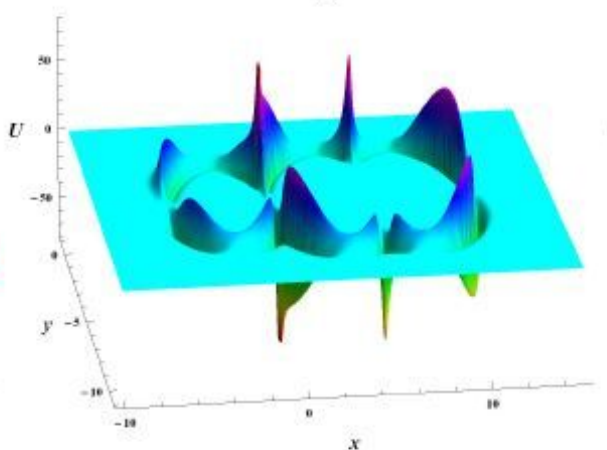

(e)

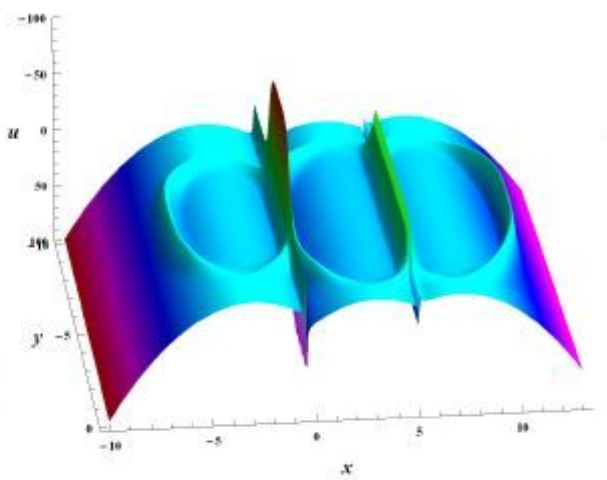

(a)
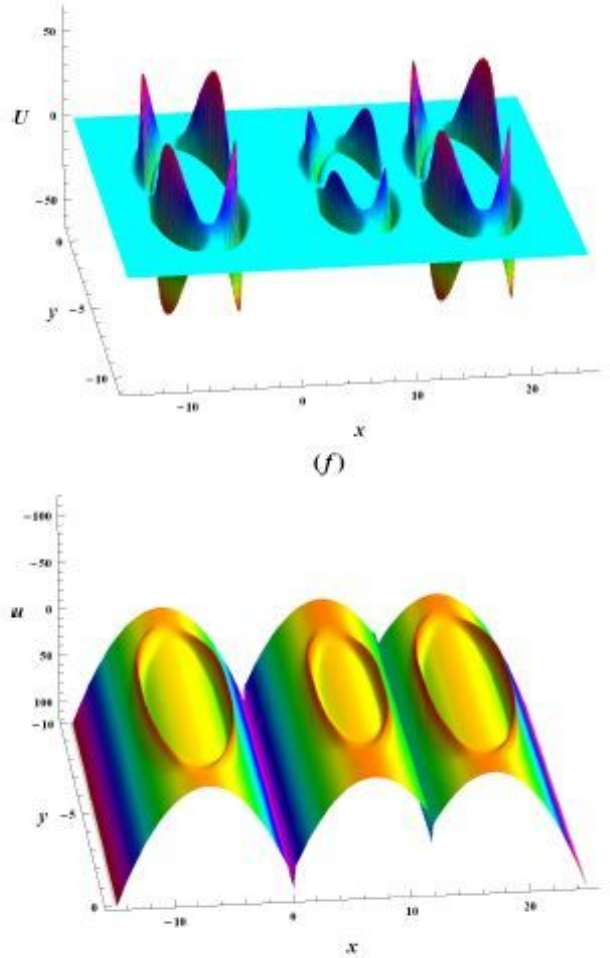

Figure 10

An ealstic interaction between an asymmetric ring soliton molecular and a ring soliton at time $(a ; d) t=-8$, $(b ; e) t=2$, and $(c ; f) t=12$ for the potential eld $U$ (upper line), and the original eld $u$ (lower line), respectively. 